Research Article

\title{
Second-Order Optimality Conditions for Set-Valued Optimization Problems Under Benson Proper Efficiency
}

\author{
Qilin Wang ${ }^{1}$ and Guolin Y $\mathbf{u}^{2}$ \\ ${ }^{1}$ College of Sciences, Chongqing Jiaotong University, Chongqing 400074, China \\ ${ }^{2}$ Research Institute of Information and System Computation Science, Beifang University of Nationalities, \\ Yinchuan 750021, China \\ Correspondence should be addressed to Qilin Wang, wangq197@126.com
}

Received 4 October 2011; Accepted 18 November 2011

Academic Editor: D. Anderson

Copyright (C) 2011 Q. Wang and G. Yu. This is an open access article distributed under the Creative Commons Attribution License, which permits unrestricted use, distribution, and reproduction in any medium, provided the original work is properly cited.

Some new properties are obtained for generalized second-order contingent (adjacent) epiderivatives of set-valued maps. By employing the generalized second-order adjacent epiderivatives, necessary and sufficient conditions of Benson proper efficient solutions are given for set-valued optimization problems. The results obtained improve the corresponding results in the literature.

\section{Introduction}

The investigation of the optimality conditions is one of the most attractive topics of optimization theory since many optimization problems encountered in economics, engineering, and other fields involve vector-valued maps (or set-valued maps) as constraints and objectives (see [1-3]). With the concept of contingent derivative for a set-valued map (see [3]), Corley [4] investigated optimality conditions for general set-valued optimization problems. But it turns out that the optimality conditions do not coincide under standard assumptions. Jahn and Rauh [5] introduced the contingent epiderivative of a set-valued map and then obtained unified necessary and sufficient optimality conditions. The essential differences between the definitions of the contingent derivative and the contingent epiderivative are that the graph is replaced by the epigraph and the derivative is single-valued. But the existence of the contingent epiderivative of a set-valued map in a general setting is an open question. To overcome the difficulty, Chen and Jahn [6] introduced a generalized contingent epiderivative of a set-valued map which is a set-valued map. They proved that the generalized contingent epiderivative exists under standard assumptions and obtained a unified necessary and sufficient condition in terms of the generalized contingent epiderivative. As to other concepts 
of epiderivatives for set-valued maps and applications to optimality conditions, one can refer to [7-11].

Since higher-order tangent sets introduced in [3], in general, are not cones and convex sets, there are some difficulties in studying higher-order optimality conditions and duality for general set-valued optimization problems. Until now, there are only a few papers to deal with higher-order optimality conditions and duality of set-valued optimization problems by virtue of the higher-order derivatives or epiderivatives introduced by the higher-order tangent sets. Jahn et al. [12] introduced second-order contingent epiderivative and generalized contingent epiderivative for a set-valued map and obtained some second-order optimality conditions based on these concepts. In [13], Li et al. studied some properties of higher-order tangent sets and higher-order derivatives introduced in [3] and then obtained higher-order Fritz John type necessary and sufficient optimality conditions for set-valued optimization problems in terms of the higher-order derivatives. By using these concepts, they also discussed higher-order Mond-Weir duality for a set-valued optimization problem in [14]. In general, since the epigraph of a set-valued map has nicer properties than the graph of a set-valued map, it is advantageous to employ the epiderivatives in set-valued optimization. Li and Chen [15] introduced the definitions of higher-order generalized contingent epiderivative and higherorder generalized adjacent epiderivative and obtained higher-order optimality conditions for Henig properly efficient solutions of a set-valued optimization problem with constraints based on the higher-order generalized adjacent epiderivative and contingent epiderivative. Chen et al. [16] introduced the concepts of higher-order weak contingent epiderivative and higher-order weak adjacent epiderivative for set-valued maps and obtained higher order Mond-Weir type duality, higher-order Wolfe type duality, and higher-order Kuhn-Tucker type optimality conditions to a constrained set-valued optimization problem based on the higher-order weak adjacent (contingent) epiderivatives and Henig efficiency. Since the existence of the higher-order contingent (adjacent) derivative and the higher-order generalized contingent (adjacent) epiderivative of a set-valued map in a general setting may not exist, Wang and Li [17] introduced the generalized higher-order contingent (adjacent) epiderivative of a set-valued map and obtained the optimality conditions for Henig efficient solutions to set-valued optimization problems. Wang et al. [18] introduced the generalized higherorder contingent (adjacent) derivatives of set-valued maps, and necessary and sufficient optimality conditions are obtained for weakly efficient solutions of set-valued optimization problems under no convexity assumptions.

To the best of our knowledge, by virtue of the second-order derivatives or epiderivatives introduced by the higher-order tangent sets, the second-order optimality conditions have not been studied for Benson proper efficient solutions of set-valued optimization problems until now. This paper aims to investigate the second-order optimality conditions for Benson proper efficient solutions of set-valued optimization problems by virtue of the generalized second-order epiderivatives.

The rest of the paper is organized as follows. In Section 2, we collect some of the concepts and introduce two kinds of set-valued optimization problem models. In Section 3, we recall the concepts and properties of generalized second-order contingent (adjacent) epiderivatives of set-valued maps and discuss some new properties of them. In Section 4, we establish second-order necessary and sufficient conditions for Benson proper efficient solutions to a set-valued optimization problem, whose constraint set is determined by a fixed set. In Section 5, we establish second-order Kuhn-Tuck type optimality conditions for Benson proper efficient solutions to a set-valued optimization problem, whose constraint set is determined by a set-valued map. 


\section{Preliminaries}

Throughout this paper, let $X, Y$, and $Z$ be three real normed spaces, where the spaces $Y$ and $Z$ are partially ordered by nontrivial closed convex pointed cones $C \subset Y$ and $D \subset Z$ with int $D \neq \emptyset$, respectively. Denote by $Y^{*}$ the topological dual space of $Y$ and by $0_{Y}$ the zero element in $Y$. The dual cone of $C$ is defined as

$$
C^{+}=\left\{f \in Y^{*} \mid f(c) \geq 0, \forall c \in C\right\}
$$

The quasi-interior $C^{+i}$ of $C^{+}$is the set

$$
C^{+i}=\left\{f \in Y^{*} \mid f(c)>0, \forall c \in C \backslash\left\{0_{Y}\right\}\right\}
$$

Recall that a base of a cone $C$ is a convex subset $B$ of $C$ such that

$$
0_{Y} \notin \mathrm{cl} B, \quad C=\text { cone } B .
$$

Of course, $C$ is pointed whenever $C$ has a base. Furthermore, if $C$ is a nonempty closed convex pointed cone in $Y$, then $C^{+i} \neq \emptyset$ if and only if $C$ has a base.

Let $F: E \rightarrow 2^{Y}$ and $G: E \rightarrow 2^{Z}$ be set-valued maps. The effective domain, the graph, and the epigraph of $F$ are defined by $\operatorname{dom}(F)=\{x \in E \mid F(x) \neq \emptyset\}, \operatorname{gph}(F)=\{(x, y) \in E \times Y \mid$ $x \in E, y \in F(x)\}$ and epi $(F)=\{(x, y) \in E \times Y \mid x \in E y \in F(x)+C\}$, respectively. Denote $F(E)=\bigcup_{x \in E} F(x)$. Let $\left(x_{0}, y_{0}\right) \in \operatorname{gph}(F), z_{0} \in G\left(x_{0}\right) \cap(-D)$.

Definition 2.1 (see [19]). $F$ is called $C$-convex on a nonempty convex subset $E$, if, for any $x_{1}$, $x_{2} \in E$ and $\lambda \in(0,1)$, such that $\lambda F\left(x_{1}\right)+(1-\lambda) F\left(x_{2}\right) \subseteq F\left(\lambda x_{1}+(1-\lambda) x_{2}\right)+C$.

Definition 2.2 (see [20]). Let $F: E \rightarrow 2^{\gamma}$ be a set-valued map, $x_{0} \in E,\left(x_{0}, y_{0}\right) \in \operatorname{gph}(F) . F$ is said to be generalized $C$-convex at $\left(x_{0}, y_{0}\right)$ on $E$, if cone (epi $\left.F-\left\{\left(x_{0}, y_{0}\right)\right\}\right)$ is convex.

Remark 2.3 (see [20]). If $F$ is $C$-convex on convex set $E$, then $F$ is generalized $C$-convex at $\left(x_{0}, y_{0}\right) \in \operatorname{gph}(F)$ on $E$. But the converse may not hold.

Definition 2.4 (see [6]). Let $M$ be a subset of $Y . y_{0} \in M$ is said to be a Benson proper efficient point of $M$ if $(-C) \cap$ clcone $\left(M+C-\left\{y_{0}\right\}\right)=\left\{0_{Y}\right\}$. The set of all Benson proper efficient points of $M$ is denoted by $P \min [M, C]$.

Definition 2.5 (see [21]). Let $Y$ be a real normed space, $B$ be a base of $C$, and $M \subset Y$ be a nonempty subset. $y \in M$ is said to be a Henig efficient point of $M$, written as $y \in \operatorname{HE}(M, B)$, if for some $\epsilon \in(0, \delta)$,

$$
(M-\{y\}) \cap-\operatorname{int}(\operatorname{cone}(\epsilon U+B))=\emptyset,
$$

where $\delta=\inf \{\|b\|: b \in B\}$ and $U$ is the closed unit ball of $Y$.

Lemma 2.6 (see [22]). Let $P$ and $C$ be two closed convex cones in a locally convex vector space, and let $C$ be pointed and have a compact base. If $P \cap(-C)=\left\{0_{Y}\right\}$, then there exists $h \in C^{+i}$ such that $h \in P^{+}$. 
Definition 2.7 (see [6]). A set-valued map $H: X \rightarrow 2^{\Upsilon}$ is said to be

(i) strictly positive homogeneous if

$$
H(\alpha x)=\alpha H(x), \quad \forall \alpha>0, \forall x \in X,
$$

(ii) subadditive if

$$
H\left(x_{1}\right)+H\left(x_{2}\right) \subseteq H\left(x_{1}+x_{2}\right)+C
$$

In this paper, consider the following set-valued optimization problem:

$$
\begin{array}{ll}
\min & F(x), \\
\text { s.t. } & x \in E .
\end{array}
$$

that is, to find a pair $\left(x_{0}, y_{0}\right)$ with $x_{0} \in K$ and $y_{0} \in F\left(x_{0}\right)$ is called a Benson proper efficient element of problem $(\mathrm{OP})$ if $y_{0} \in P \min [F(E), C]$. We also consider the following constraint set-valued optimization problem:

$$
\begin{array}{ll}
\min & F(x), \\
\text { s.t. } & G(x) \cap(-D) \neq \emptyset, \quad x \in E .
\end{array}
$$

Set $K:=\{x \in E \mid G(x) \cap(-D) \neq \emptyset\}$. A pair $\left(x_{0}, y_{0}\right)$ with $x_{0} \in K$ and $y_{0} \in F\left(x_{0}\right)$ is called a Benson proper efficient element of problem (SOP) if $y_{0} \in P \min [F(K), C]$.

\section{Properties of Generalized Second-Order Contingent (Adjacent) Epiderivatives}

In this section, we discuss some new properties of generalized second-order contingent and adjacent epiderivatives. Moreover, we give an example to show these properties. Let $F$ be a set-valued map from $E \subseteq X$ to $Y$.

Definition 3.1 (see [17]). Let $F$ be a set-valued map from $E \subseteq X$ to $Y,\left(x_{0}, y_{0}\right) \in \operatorname{gph}(H)$, $u \in X, v \in Y$.

(i) The generalized second-order contingent epiderivative $G-D^{(2)} F\left(x_{0}, y_{0}, u, v\right)$ of $F$ at $\left(x_{0}, y_{0}\right)$ for vectors $(u, v)$ is the set-valued map from $X$ to $Y$ defined by

$$
\operatorname{gph}\left(G-D^{(2)} F\left(x_{0}, y_{0}, u, v\right)\right)=G-T_{\mathrm{epi}(F)}^{(2)}\left(x_{0}, y_{0}, u, v\right)
$$

(ii) The generalized second-order adjacent epiderivative $G-D^{b(2)} F\left(x_{0}, y_{0}, u, v\right)$ of $F$ at $\left(x_{0}, y_{0}\right)$ for vectors $(u, v)$ is the set-valued map from $X$ to $Y$ defined by

$$
\operatorname{gph}\left(G-D^{b(2)} F\left(x_{0}, y_{0}, u, v\right)\right)=G-T_{\mathrm{epi}(F)}^{b(2)}\left(x_{0}, y_{0}, u, v\right)
$$


Remark 3.2. If $(u, v)=\left(0_{X}, 0_{Y}\right)$, then

$$
\begin{aligned}
G-D^{(2)} F\left(x_{0}, y_{0}, u, v\right) & =G-D F\left(x_{0}, y_{0}\right), \\
G-D^{b(2)} F\left(x_{0}, y_{0}, u, v\right) & =G-D^{b} F\left(x_{0}, y_{0}\right) .
\end{aligned}
$$

From [17, Proposition 3.9 and Corollary 3.11], we have the following result.

Proposition 3.3. Let $x, x_{0} \in E, y_{0} \in F\left(x_{0}\right),(u, v) \in\left\{0_{X}\right\} \times C$. Then,

(i) $F(x)+C-\left\{y_{0}\right\} \subset G-D^{(2)} F\left(x_{0}, y_{0}, u, v\right)\left(x-x_{0}\right)$;

(ii) $F(x)+C-\left\{y_{0}\right\} \subset G-D^{b(2)} F\left(x_{0}, y_{0}, u, v\right)\left(x-x_{0}\right)$.

Proposition 3.4. Let $E$ be a nonempty subset of $X, x_{0} \in E, y_{0} \in F\left(x_{0}\right)$. Let $F$ be generalized $C$ convex at $\left(x_{0}, y_{0}\right)$ on $E, u \in E, v \in F(u)+C$. Then,

$$
F(x)+C-\left\{y_{0}\right\} \subset G-D^{b(2)} F\left(x_{0}, y_{0}, u-x_{0}, v-y_{0}\right)\left(x-x_{0}\right), \quad \text { for any } x \in E .
$$

Proof. Take a arbitrary sequence $\left\{h_{n}\right\}$ with $h_{n} \rightarrow 0^{+}$, any $c \in C, x \in E$, and $y \in F(x)$. Since $H$ is generalized $C$-convex at $\left(x_{0}, y_{0}\right)$ on $E$, cone $\left(\operatorname{epi}(F)-\left\{\left(x_{0}, y_{0}\right)\right\}\right)$ is convex, and then

$$
h_{n}\left(u-x_{0}, v-y_{0}\right) \in \text { cone }\left(\text { epi } F-\left\{\left(x_{0}, y_{0}\right)\right\}\right)
$$

It follows from $h_{n}>0$, and cone(epi $\left.F-\left\{\left(x_{0}, y_{0}\right)\right\}\right)$ is a convex cone that

$$
\left(x_{n}, y_{n}\right):=h_{n}\left(u-x_{0}, v-y_{0}\right)+h_{n}^{2}\left(x-x_{0}, y+c-y_{0}\right) \in \text { cone }\left(\text { epi } F-\left\{\left(x_{0}, y_{0}\right)\right\}\right)
$$

Then,

$$
\left(x-x_{0}, y+c-y_{0}\right)=\frac{\left(x_{n}, y_{n}\right)-h_{n}\left(u-x_{0}, v-y_{0}\right)}{h_{n}^{2}}
$$

Thus,

$$
F(x)+C-\left\{y_{0}\right\} \subset G-D^{b(2)} F\left(x_{0}, y_{0}, u-x_{0}, v-y_{0}\right)\left(x-x_{0}\right),
$$

and the proof of the proposition is complete.

Corollary 3.5. Let $E$ be a nonempty subset of $X, x_{0} \in E, y_{0} \in F\left(x_{0}\right)$. If $F$ is generalized C-convex at $\left(x_{0}, y_{0}\right)$ on $E, u \in E, v \in F(u)+C$, then

$$
F(x)+C-\left\{y_{0}\right\} \subset G-D^{(2)} F\left(x_{0}, y_{0}, u-x_{0}, v-y_{0}\right)\left(x-x_{0}\right), \quad \text { for any } x \in E .
$$


Proposition 3.6. Let $\left(x_{0}, y_{0}\right) \in \operatorname{gph}(F),(u, v) \in X \times Y$. Then,

(i) G-D $D^{b(2)} F\left(x_{0}, y_{0}, u, v\right)$ is strictly positive homogeneous.

Moreover, if $F$ is generalized $C$-convex at $\left(x_{0}, y_{0}\right)$ on $E$, then

(ii) $G-D^{b(2)} F\left(x_{0}, y_{0}, u, v\right)$ is subadditive.

Proof. (i) Since

$$
G-T_{\mathrm{epi}(F)}^{b(2)}\left(x_{0}, y_{0}, u, v\right)=T_{\text {cone }\left(\operatorname{epi}(F)-\left\{\left(x_{0}, y_{0}\right)\right\}\right)}^{b(2)}\left(0_{X}, 0_{Y}, u, v\right)=T_{\text {cone }\left(\operatorname{epi}(F)-\left\{\left(x_{0}, y_{0}\right)\right\}\right)}^{b}(u, v),
$$

$G-D^{b(2)} F\left(x_{0}, y_{0}, u, v\right)$ is strictly positive homogeneous.

(ii) Let $x_{1}, x_{2} \in X, y_{1} \in G-D^{b(2)} F\left(x_{0}, y_{0}, u, v\right)\left(x_{1}\right), y_{2} \in G-D^{b(2)} F\left(x_{0}, y_{0}, u, v\right)\left(x_{2}\right)$. Then one has $\left(x_{1}, y_{1}\right),\left(x_{2}, y_{2}\right) \in G-T_{\text {epi }(F)}^{b(2)}\left(x_{0}, y_{0}, u, v\right)$. Since $F$ is generalized $C$-convex at $\left(x_{0}, y_{0}\right)$ on $E$, cone (epi $\left.F-\left\{\left(x_{0}, y_{0}\right)\right\}\right)$ is convex, and then it follows from the proof of [13, Proposition 3.2], that $G-T_{\operatorname{epi}(F)}^{b(2)}\left(x_{0}, y_{0}, u, v\right)$ is convex. Thus, we have

$$
\frac{1}{2}\left(x_{1}, y_{1}\right)+\frac{1}{2}\left(x_{2}, y_{2}\right)=\frac{1}{2}\left(x_{1}+x_{2}, y_{1}+y_{2}\right) \in G-T_{\mathrm{epi}(F)}^{b(2)}\left(x_{0}, y_{0}, u, v\right),
$$

and then it follows from (i) that

$$
y_{1}+y_{2} \in G-D^{b(2)} F\left(x_{0}, y_{0}, u, v\right)\left(x_{1}+x_{2}\right),
$$

which implies

$$
\begin{gathered}
G-D^{b(2)} F\left(x_{0}, y_{0}, u, v\right)\left(x_{1}\right)+G-D^{b(2)} F\left(x_{0}, y_{0}, u, v\right)\left(x_{2}\right) \\
\subseteq G-D^{b(2)} F\left(x_{0}, y_{0}, u, v\right)\left(x_{1}+x_{2}\right)+C .
\end{gathered}
$$

The proof of the proposition is complete.

For the sake of comparison, we recall some notions in $[13,15,16]$.

Definition 3.7 (see [13]). The second-order adjacent derivative $D^{b(2)} F\left(x_{0}, y_{0}, u, v\right)$ of $F$ at $\left(x_{0}, y_{0}\right)$ for vector $(u, v)$ is the set-valued map from $X$ to $Y$ defined by

$$
\operatorname{gph}\left(D^{b(2)} F\left(x_{0}, y_{0}, u, v\right)\right)=T_{\operatorname{Graph}(F)}^{b(2)}\left(x_{0}, y_{0}, u, v\right)
$$

Definition 3.8 (see [15]). The second-order generalized adjacent epiderivative $D_{g}^{b(2)} F\left(x_{0}\right.$, $\left.y_{0}, u, v\right)$ of $F$ at $\left(x_{0}, y_{0}\right)$ for vectors $(u, v)$ is the set-valued map from $X$ to $Y$ defined by

$$
D_{g}^{b(2)} F\left(x_{0}, y_{0}, u, v\right)(x)=\operatorname{Min}_{C}\left\{y \in Y:(x, y) \in T_{\mathrm{epi}(F)}^{b(2)}\left(x_{0}, y_{0}, u, v\right)\right\}
$$


Definition 3.9 (see [16]). The second-order weak adjacent epiderivative $D_{g}^{b(2)} F\left(x_{0}, y_{0}, u, v\right)$ of $F$ at $\left(x_{0}, y_{0}\right)$ for vector $(u, v)$ is the set-valued map from $X$ to $Y$ defined by

$$
D_{w}^{b(2)} F\left(x_{0}, y_{0}, u, v\right)(x)=W \operatorname{Min}_{C}\left\{y \in Y:(x, y) \in T_{\mathrm{epi}(F)}^{b(2)}\left(x_{0}, y_{0}, u, v\right)\right\}
$$

Remark 3.10. Notice that in Proposition 3.6, we establish a special property of $G-D^{b(2)} F\left(x_{0}, y_{0}\right.$, $u, v)$, which is similar to the corresponding property of the generalized contingent epiderivative in [6, Theorem 1]. But we cannot obtain the similar property for $D^{b(2)} F\left(x_{0}, y_{0}, u, v\right)$, $D_{g}^{b(2)} F\left(x_{0}, y_{0}, u, v\right)$, and $D_{w}^{b(2)} F\left(x_{0}, y_{0}, u, v\right)$, even though $F$ is a $C$-convex map. The following example explains the case.

Example 3.11. Let $X=R, Y=R^{2}, C=R_{+}^{2}, F(x)=\left\{\left(y_{1}, y_{2}\right) \in R^{2} \mid y_{1} \geq x, y_{2} \geq x^{2}\right\}, \forall x \in X$. Take $\left(x_{0}, y_{0}\right)=(0,(0,0)) \in \operatorname{gph}(F)$ and $(u, v)=(1,(1,0))$. Then,

$$
\begin{gathered}
T_{\mathrm{gph}(F)}^{b(2)}\left(x_{0}, y_{0}, u, v\right)=T_{\mathrm{epi}(F)}^{b(2)}\left(x_{0}, y_{0}, u, v\right)=\bigcup_{x \in R}(\{x\} \times[x,+\infty) \times[1,+\infty)), \\
G-T_{\mathrm{epi}(F)}^{b(2)}\left(x_{0}, y_{0}, u, v\right)=\bigcup_{x \in R}\left(\{x\} \times[x,+\infty) \times R_{+}\right) .
\end{gathered}
$$

Therefore, for any $x \in X$, one has

$$
\begin{gathered}
D^{b(2)} F\left(x_{0}, y_{0}, u, v\right)(x)=[x,+\infty) \times[1,+\infty), \\
D_{g}^{b(2)} F\left(x_{0}, y_{0}, u, v\right)(x)=\{(x, 1)\}, \\
D_{w}^{b(2)} F\left(x_{0}, y_{0}, u, v\right)(x)=\left\{\left(x, y_{2}\right) y_{2} \geq 1\right\} \cup\left\{\left(y_{1}, 1\right) \mid y_{1} \geq x\right\}, \\
G-D^{b(2)} F\left(x_{0}, y_{0}, u, v\right)(x)=[x,+\infty) \times R_{+} .
\end{gathered}
$$

Naturally, $D^{b(2)} F\left(x_{0}, y_{0}, u, v\right), D_{g}^{b(2)} F\left(x_{0}, y_{0}, u, v\right)$, and $D_{w}^{b(2)} F\left(x_{0}, y_{0}, u, v\right)$ are not strictly positive homogeneous, but $G-D^{b(2)} F\left(x_{0}, y_{0}, u, v\right)$ is strictly positive homogeneous here.

It follows from [13, Corollary 3.1], and the proof of Proposition 3.6 that the following result holds.

Corollary 3.12. Let $\left(x_{0}, y_{0}\right) \in \operatorname{gph}(F),(u, v) \in X \times Y$. Then

(i) $G-D^{(2)} F\left(x_{0}, y_{0}, u, v\right)$ is strictly positive homogeneous.

Moreover, if $F$ is generalized $C$-convex at $\left(x_{0}, y_{0}\right)$ on $E$, and $(u, v) \in$ cone(epi $(H)-$ $\left.\left\{\left(x_{0}, y_{0}\right)\right\}\right)$, then

(ii) $G-D^{(2)} F\left(x_{0}, y_{0}, u, v\right)$ is subadditive.

Remark 3.13. Under the assumption of cone-convex maps, Chen and Jahn [6, Theorem 1] obtained the subadditivity of $D_{g} F\left(x_{0}, y_{0}\right)$. However, it is under the assumption of generalized cone-convex maps that we obtain the subadditivity of $G-D^{(2)} F\left(x_{0}, y_{0}, u, v\right)$ in Corollary 3.12.

By Propositions 3.3-3.6 and Corollary 3.12, we obtain the following results. 
Corollary 3.14. Let $\left(x_{0}, y_{0}\right) \in \operatorname{gph}(F),(u, v) \in X \times Y$. Then,

$$
\begin{aligned}
& \left(0_{X}, 0_{Y}\right) \in \operatorname{gph}\left(G-D^{(2)} F\left(x_{0}, y_{0}, u, v\right)\right), \\
& \left(0_{X}, 0_{Y}\right) \in \operatorname{gph}\left(G-D^{b(2)} F\left(x_{0}, y_{0}, u, v\right)\right) .
\end{aligned}
$$

Corollary 3.15. Let $\left(x_{0}, y_{0}\right) \in \operatorname{gph}(F)$ and $(u, v) \in X \times Y$. If $F$ is generalized C-convex at $\left(x_{0}, y_{0}\right)$ on E, then

(i) $\operatorname{gph}\left(G-D^{b(2)} F\left(x_{0}, y_{0}, u, v\right)\right)$ is a closed convex cone.

Moreover, if $(u, v) \in$ cone $\left(\operatorname{epi}(H)-\left\{\left(x_{0}, y_{0}\right)\right\}\right)$, then

(ii) $\operatorname{gph}\left(G-D^{(2)} F\left(x_{0}, y_{0}, u, v\right)\right)$ is a closed convex cone.

\section{Second-Order Optimality Conditions of (OP)}

In this section, by employing the generalized second-order adjacent epiderivative, we will discuss the second-order optimality conditions for Benson proper efficient solutions of (OP).

Theorem 4.1. Let $x_{0} \in E, y_{0} \in F\left(x_{0}\right)$, and $(u, v) \in X \times(-C)$. If $\left(x_{0}, y_{0}\right)$ is a Benson proper efficient element of (OP), then, for every $x \in \bar{\Omega}:=\operatorname{dom}\left[G-D^{b(2)} F\left(x_{0}, y_{0}, u, v\right)\right]$, one has

$$
G-D^{b(2)} F\left(x_{0}, y_{0}, u, v\right)(x) \subset Y \backslash\left(-C \backslash\left\{0_{Y}\right\}\right) .
$$

Proof. Since $\left(x_{0}, y_{0}\right)$ is a Benson proper efficient element of $(\mathrm{OP})$,

$$
\text { clcone }\left(F(E)+C-\left\{y_{0}\right\}\right) \cap(-C)=\left\{0_{Y}\right\}
$$

Assume that there exists an $\bar{x} \in \bar{\Omega}$ such that (4.1) does not hold, that is, there exists

$$
\bar{y} \in G-D^{b(2)} F\left(x_{0}, y_{0}, u, v\right)(\bar{x}) \cap\left(-C \backslash\left\{0_{Y}\right\}\right),
$$

and then, by the definition of generalized second-order adjacent epiderivatives, for arbitrary sequence $\left\{h_{n}\right\}$ with $h_{n} \rightarrow 0^{+}$, there exists a sequence

$$
\left\{\left(x_{n}, y_{n}\right)\right\} \in \operatorname{cone}\left(\operatorname{epi} F-\left\{\left(x_{0}, y_{0}\right)\right\}\right)
$$

such that

$$
\frac{\left(x_{n}, y_{n}\right)-h_{n}(u, v)}{h_{n}^{2}} \longrightarrow(\bar{x}, \bar{y})
$$

Since $v \in-C, h_{n}>0$ and $C$ is a convex cone, $h_{n} v \in-C$. Then, by (4.4), we get

$$
y_{n}-h_{n} v \in \operatorname{cone}\left(F(E)+C-\left\{y_{0}\right\}\right) \text {. }
$$


Thus, from (4.5) and (4.3), one obtains

$$
\bar{y} \in \operatorname{clcone}\left(F(E)+C-\left\{y_{0}\right\}\right) \cap\left(-C \backslash\left\{0_{Y}\right\}\right),
$$

which contradicts (4.2). So (4.1) holds and the proof is complete.

Theorem 4.2. Let $(u, v) \in X \times(-C), x_{0} \in E, y_{0} \in F\left(x_{0}\right)$ and let $C$ be locally compact. Suppose that the following conditions are satisfied:

(i) $F$ is generalized C-convex at $\left(x_{0}, y_{0}\right)$ on $E$;

(ii) the pair $\left(x_{0}, y_{0}\right)$ is a Benson proper efficient element of (OP).

Then, there exists $\phi \in C^{+i}$ such that

$$
\inf \left\{\phi(y) \mid y \in \bigcup_{x \in \bar{\Omega}} G-D^{b(2)} F\left(x_{0}, y_{0}, u, v\right)(x)\right\}=0,
$$

where $\bar{\Omega}:=\operatorname{dom}\left[G-D^{b(2)} F\left(x_{0}, y_{0}, u, v\right)\right]$.

Proof. Define

$$
M=\bigcup_{x \in \bar{\Omega}} G-D^{b(2)} F\left(x_{0}, y_{0}, u, v\right)(x)
$$

By the similar line of proof for convexity of $M$ in [15], Theorem 5.1, we obtain that $M$ is a convex set. It follows from Theorem 4.1, that

$$
M \cap(-C)=\left\{0_{Y}\right\}
$$

Thus, by Lemma 2.6, there exists $\phi \in C^{+i}$ such that $\phi \in M^{+}$. So, we have

$$
\phi(y) \geq 0, \quad \forall y \in M
$$

It follows from Corollary 3.15 that $0_{Y} \in M$, so

$$
\inf \left\{\phi(y) \mid y \in \bigcup_{x \in \bar{\Omega}} G-D^{b(2)} F\left(x_{0}, y_{0}, u, v\right)(x)\right\}=0,
$$

and the proof of the theorem is complete.

Theorem 4.3. Let $x_{0} \in E, y_{0} \in F\left(x_{0}\right)$ and $(u, v) \in\left\{0_{X}\right\} \times C$. If there exists $\phi \in C^{+i}$ such that

$$
\phi(y) \geq 0, \quad \forall y \in G-D^{b(2)} F\left(x_{0}, y_{0}, u, v\right)\left(x-x_{0}\right), \quad x \in E,
$$

then the pair $\left(x_{0}, y_{0}\right)$ is a Benson proper efficient solution of (OP). 
Proof. It follows from Proposition 3.3 that

$$
F(x)+C-\left\{y_{0}\right\} \subset G-D^{b(2)} F\left(x_{0}, y_{0}, u, v\right)\left(x-x_{0}\right),
$$

for all $x \in E, y \in F(x)$. Then, by (4.13), (4.14), and $\phi \in C^{+i}$, we get

$$
\phi(y) \geq \phi\left(y_{0}\right), \quad \forall y \in F(E)
$$

Thus, by the sufficient condition of [23, Theorem 4.1], we get that $\left(x_{0}, y_{0}\right)$ is a Benson proper efficient solution of $(\mathrm{OP})$, and the proof of the theorem is complete.

Remark 4.4. In Theorem 4.3, no assumption of generalized convexity is imposed.

Remark 4.5. If we use $D^{b(2)} F\left(x_{0}, y_{0}, u, v\right)$ instead of $G-D^{b(2)} F\left(x_{0}, y_{0}, u, v\right)$ in Theorem 4.3, then the corresponding result for $D^{b(2)} F\left(x_{0}, y_{0}, u, v\right)$ may not hold. The following example explains the case.

Example 4.6. Suppose that $X=Y=E=R, C=R_{+}$. Let $F: E \rightarrow 2^{Y}$ be a set-valued map with

$$
F(x)=\left\{\begin{array}{l}
\left\{y \in R: y \geq|x|+1, x \leq \frac{1}{2}\right\}, \\
\left\{y \in R: y \geq-x+1, x>\frac{1}{2}\right\} .
\end{array}\right.
$$

Consider the following constrained set-valued optimization problem (4.1):

$$
\begin{array}{ll}
\min & F(x), \\
\text { s.t. } & x \in E .
\end{array}
$$

Take $\left(x_{0}, y_{0}\right)=(0,1) \in \operatorname{gph}(F),(u, v)=(0,0) \in\{0\} \times C$. We have

$$
D^{b(2)} F\left(x_{0}, y_{0}, u, v\right)\left(x-x_{0}\right)=\{y \in R: y \geq|x|\}, \quad \forall x \in E .
$$

Then, for any $\phi \in C^{+i}$, we have

$$
\phi(y) \geq 0, \quad \forall y \in D^{b(2)} F\left(x_{0}, y_{0}, u, v\right)\left(x-x_{0}\right), \quad x \in E .
$$

Naturally, the pair $\left(x_{0}, y_{0}\right)$ is not a Benson proper efficient solution of (4.1).

From Proposition 3.4, we know that the following theorem holds.

Theorem 4.7. Let $x_{0} \in E, y_{0} \in F\left(x_{0}\right)$. Suppose that the following conditions are satisfied:

(i) $u \in E, v \in F(u)+C$,

(ii) $F$ is generalized $C$-convex at $\left(x_{0}, y_{0}\right)$ on $E$, 
(iii) there exists $\phi \in C^{+i}$ such that

$$
\phi(y) \geq 0, \quad \forall y \in G-D^{b(2)} F\left(x_{0}, y_{0}, u-x_{0}, v-y_{0}\right)\left(x-x_{0}\right), x \in E .
$$

Then the pair $\left(x_{0}, y_{0}\right)$ is a Benson proper efficient solution of $(\mathrm{OP})$.

\section{Second-Order Kuhn-Tuck Type Optimality Conditions of (SOP)}

In this section, by employing the generalized second-order adjacent epiderivative, we will discuss the second-order Kuhn-Tuck type optimality conditions for Benson proper efficient solutions of (SOP). The notation $(F, G)(x)$ is used to denote $F(x) \times G(x)$. Let $x_{0} \in K, y_{0} \in$ $F\left(x_{0}\right)$. Firstly, we recall a result in [24].

The interior tangent cone of $K$ at $x_{0}$ is defined as

$$
\operatorname{IT}_{K}\left(x_{0}\right)=\left\{u \in X \exists \lambda>0, \forall t \in(0, \lambda), \forall u^{\prime} \in B_{X}(u, \lambda), x_{0}+t u^{\prime} \in K\right\},
$$

where $B_{X}(u, \lambda)$ stands for the closed ball centered at $u \in X$ and of radius $\lambda$.

Lemma 5.1 (see [24]). If $K \subset X$ is convex, $x_{0} \in K$ and int $K \neq \emptyset$, then

$$
\operatorname{IT}_{\text {int } K}\left(x_{0}\right)=\text { int cone }\left(K-\left\{x_{0}\right\}\right) .
$$

Theorem 5.2. Let $(u, v, w) \in X \times(-C) \times(-D), z_{0} \in G\left(x_{0}\right) \cap(-D)$. If $\left(x_{0}, y_{0}\right)$ is a Benson proper efficient element of $(C P)$, then

$$
\left[G-D^{b(2)}(F, G)\left(x_{0}, y_{0}, z_{0}, u, v, w_{1}+z_{0}\right)(x)\right] \subset(Y \times Z) \backslash\left(-\left(\left(C \backslash\left\{0_{Y}\right\}\right) \times\left(\operatorname{int} D+z_{0}\right)\right)\right),
$$

for all $x \in \Omega:=\operatorname{dom}\left[G-D^{b(2)}(F, G)\left(x_{0}, y_{0}, z_{0}, u, v, w_{1}+z_{0}\right)\right]$.

Proof. Since $\left(x_{0}, y_{0}\right)$ is a Benson proper efficient element of $(C P)$,

$$
\operatorname{clcone}\left(F(K)+C-\left\{y_{0}\right\}\right) \cap-C=\left\{0_{Y}\right\} .
$$
some

Suppose that there exists some $\bar{x} \in \Omega$ such that (5.3) does not hold, that is, there exists

$$
\begin{gathered}
(\bar{y}, \bar{z}) \in G-D^{b(2)}(F, G)\left(x_{0}, y_{0}, z_{0}, u, v, w+z_{0}\right)(\bar{x}) \\
(\bar{y}, \bar{z}) \in-\left(\left(C \backslash\left\{0_{Y}\right\}\right) \times\left(\operatorname{int} D+\left\{z_{0}\right\}\right)\right) .
\end{gathered}
$$

It follows from (5.5) and the definition of generalized second-order contingent epiderivatives that there exist sequences $\left\{h_{n}\right\}$ with $h_{n} \rightarrow 0^{+}$and

$$
\left\{\left(x_{n}, y_{n}, z_{n}\right)\right\} \in \operatorname{cone}\left(\operatorname{epi}(F, G)-\left\{\left(x_{0}, y_{0}, z_{0}\right)\right\}\right)
$$


such that

$$
\frac{\left(x_{n}, y_{n}, z_{n}\right)-h_{n}\left(u, v, w+z_{0}\right)}{h_{n}^{2}} \longrightarrow(\bar{x}, \bar{y}, \bar{z})
$$

From (5.6) and (5.8), there exists a sufficiently large natural number $N_{1}$ such that

$$
\begin{aligned}
\bar{z}_{n}: & =\frac{z_{n}-h_{n}\left(w+z_{0}\right)}{h_{n}^{2}} \\
& =\frac{1}{h_{n}}\left(\frac{z_{n}-h_{n} w}{h_{n}}-z_{0}\right) \\
& \in-\left(\operatorname{int} D+z_{0}\right) \subset-\operatorname{int} \operatorname{cone}\left(D+\left\{z_{0}\right\}\right), \quad \forall n>N_{1} .
\end{aligned}
$$

According to (5.9) and Lemma 5.1, we obtain $-\bar{z} \in \operatorname{IT}_{\text {int } D}\left(-z_{0}\right)$. Then, it follows from the definitions of $\operatorname{IT}_{\operatorname{int} D}\left(-z_{0}\right)$ that $\exists \lambda>0, \forall t \in(0, \lambda), \forall u^{\prime} \in B_{Z}(-\bar{z}, \lambda),-z_{0}+t u^{\prime} \in \operatorname{int} D$. Since $h_{n} \rightarrow 0^{+}$and (5.9), there exists a sufficiently large natural number $N_{2}$ such that $h_{n} \in(0, \lambda)$,for $n>N_{2}$ and $-z_{0}+h_{n}\left(-\bar{z}_{n}\right) \in \operatorname{int} D$, for $n>N_{2}$, that is,

$$
\frac{z_{n}-h_{n} w}{h_{n}} \in-\operatorname{int} D, \quad \text { for } n>N_{2}
$$

It follows from $h_{n}>0, w \in-D$ and $D$ is a convex cone that

$$
z_{n} \in-\operatorname{int} D, \quad \text { for } n>N_{2} \text {. }
$$

Then from (5.7), $z_{n} \in \operatorname{cone}\left(G\left(x_{n}\right)+D-\left\{z_{0}\right\}\right)$, there exist $\lambda_{n}>0, \tilde{x}_{n} \in E, \tilde{z}_{n} \in G\left(\tilde{x}_{n}\right), \tilde{y}_{n} \in$ $F\left(\tilde{x}_{n}\right), c_{n} \in C$ and $d_{n} \in D$ such that $\left.y_{n}=\lambda_{n}\left(\tilde{y}_{n}+c_{n}-y_{0}\right)\right)$ and $\left.z_{n}=\lambda_{n}\left(\tilde{z}_{n}+d_{n}-z_{0}\right)\right)$. It follows from (5.11) that $\tilde{z}_{n} \in G\left(\tilde{x}_{n}\right) \cap(-D)$, for $n>N_{2}$, and then

$$
\tilde{x}_{n} \in K, \quad \text { for } n>N_{2} \text {. }
$$

Since $v \in-C, h_{n}>0$ and $C$ is a convex cone, $h_{n} v \in-C$. Then by (5.7), (5.12), and (5.8), we get

$$
\bar{y} \in \operatorname{clcone}\left(F(K)+C-\left\{y_{0}\right\}\right), \quad \text { for } n>\max \left\{N_{1}, N_{2}\right\}
$$

Then it follows from (5.6) that

$$
\bar{y} \in \operatorname{clcone}\left(F(K)+C-\left\{y_{0}\right\}\right) \cap-\left(C \backslash\left\{0_{Y}\right\}\right), \quad \text { for } n>\max \left\{N_{1}, N_{2}\right\},
$$

which contradicts (5.4). Thus, (5.3) holds and the proof of the theorem is complete. 
Theorem 5.3. Let $(u, v, w) \in X \times(-C) \times(-D), x_{0} \in E,\left(y_{0}, z_{0}\right) \in(F, G)\left(x_{0}\right)$. Suppose that the following conditions are satisfied:

(i) C has a compact base;

(ii) $(F, G)$ is generalized $C \times D$-convex at $\left(x_{0}, y_{0}, z_{0}\right)$ on $E$;

(iii) the pair $\left(x_{0}, y_{0}\right)$ is a Benson proper efficient element of (SOP).

Then, there exist $\phi \in C^{+i}$ and $\psi \in D^{*}$ such that

$$
\inf \{\phi(y)+\psi(z) \mid(y, z) \in \Delta\}=0, \quad \psi\left(z_{0}\right)=0,
$$

where $\Delta:=\bigcup_{x \in \Omega} G-D^{b(2)}(F, G)\left(x_{0}, y_{0}, z_{0}, u, v, w+z_{0}\right)(x)$ and $\Omega:=\operatorname{dom}\left[G-D^{b(2)}(F, G)\left(x_{0}\right.\right.$, $\left.\left.y_{0}, z_{0}, u, v, w+z_{0}\right)\right]$.

Proof. Define

$$
\bar{M}=\bigcup_{x \in \Omega}\left[G-D^{b(2)}(F, G)\left(x_{0}, y_{0}, z_{0}, u, v, w+z_{0}\right)(x)+\left(0_{Y}, z_{0}\right)\right] .
$$

By the similar line of proof for convexity of $M$ in [15, Theorem 5.1], we obtain that $\bar{M}$ is a convex set. It follows from Theorem 5.2 that

$$
\bar{M} \cap\left(-\left(\left(C \backslash\left\{0_{Y}\right\}\right) \times \operatorname{int} D\right)\right)=\emptyset .
$$

Thus, by employing Corollary 3.15, it follows from a standard separation theorem of convex sets and the similar proof method of [8, Theorem 1] that there exist $\phi \in C^{+i}$ and $\psi \in D^{*}$ such that

$$
\inf \{\phi(y)+\psi(z) \mid(y, z) \in \Delta\}=0, \quad \psi\left(z_{0}\right)=0 .
$$

The proof is complete.

Remark 5.4. It follows from Remarks 2.3 and 3.2 that the necessary optimality condition in Theorem 5.3 is obtained under weaker assumptions than those assumed of [8, Theorem 1].

Now we give an example to illustrate the necessary optimality conditions for generalized second-order contingent epiderivatives.

Example 5.5. Suppose that $X=Y=Z=E=R, C=D=R_{+}$. Let $F: E \rightarrow 2^{Y}$ be a set-valued map with

$$
F(x)=\left\{y \in R \mid y \geq x^{2 / 3}\right\}, \quad x \in E,
$$

and $G: E \rightarrow Z$ be a set-valued map with

$$
G(x)=\{z \in R \mid z \geq-x\}, \quad x \in E .
$$


Consider the following constrained set-valued optimization problem (4.2):

$$
\begin{aligned}
\min & F(x), \\
\text { s.t. } & x \in E, \quad G(x) \cap(-D) \neq \emptyset .
\end{aligned}
$$

Take $\left(x_{0}, y_{0}, z_{0}\right)=(0,0,0) \in \operatorname{gph}(F, G)$ and $(u, v, w)=(1,0,-1) \in X \times(-C) \times(-D)$. Naturally, $(F, G)$ is generalized $C \times D$-convex at $\left(x_{0}, y_{0}, z_{0}\right)$ on $E$, and $\left(x_{0}, y_{0}\right)$ is a Benson proper efficient solution of (4.2). By directly calculation, we have

$$
G-D^{b(2)}(F, G)\left(x_{0}, y_{0}, z_{0}, u_{1}, v_{1}, w_{1}+z_{0}\right)(x)=\left\{(y, z) \in R^{2}: y \geq 0, z \geq-x\right\}, \quad \forall x \in E .
$$

Take $\phi=1 \in C^{+i}$ and $\varphi=0 \in D^{*}$. Naturally, necessary optimality condition of Theorem 5.3 holds here.

Theorem 5.6. Let $x_{0} \in E,\left(y_{0}, z_{0}\right) \in(F, G)\left(x_{0}\right)$. Suppose that the following conditions are satisfied:

(i) $(u, v, w) \in\left\{0_{X}\right\} \times C \times D$,

(ii) there exist $\phi \in C^{+i}$ and $\psi \in D^{*}$ such that

$$
\begin{aligned}
& \qquad \inf \{\phi(y)+\psi(z) \mid(y, z) \in \Theta\}=0, \quad \psi\left(z_{0}\right)=0, \\
& \text { where } \Theta:=\bigcup_{x \in K} G-D^{b(2)}(F, G)\left(x_{0}, y_{0}, z_{0}, u, v, w\right)\left(x-x_{0}\right) .
\end{aligned}
$$

Then, the pair $\left(x_{0}, y_{0}\right)$ is a Benson proper efficient solution of (SOP).

Proof. It follows from Proposition 3.3 that

$$
\left(y-y_{0}, z-z_{0}\right) \in G-D^{b(2)}(F, G)\left(x_{0}, y_{0}, z_{0}, u, v, w\right)\left(x-x_{0}\right),
$$

for all $y \in F(x), z \in G(x), x \in K$. Then, by assumption (ii), we have

$$
\phi\left(y-y_{0}\right)+\psi\left(z-z_{0}\right) \geq 0, \quad \forall y \in F(K), z \in G(K) .
$$

Thus, there exists a $\bar{z} \in G(K)$ with $\bar{z} \in-D$ such that $\psi(\bar{z}) \leq 0$. It follows from $\psi\left(z_{0}\right)=0$ and (5.25) that

$$
\phi(y) \geq \phi\left(y_{0}\right), \quad \forall y \in F(K) .
$$

It follows from the sufficient condition of [23, Theorem 4.1], that $\left(x_{0}, y_{0}\right)$ is a Benson proper efficient solution of (SOP) and the proof of the theorem is complete.

Remark 5.7. Since Theorem 5.6 does not involve the assumption of convexity, it improves and generalizes [8, Theorem 2]. 
From Proposition 3.4 and the proof of Theorem 5.6, we easily obtain that the following Theorem holds.

Theorem 5.8. Let $x_{0} \in E,\left(y_{0}, z_{0}\right) \in(F, G)\left(x_{0}\right)$. Suppose that the following conditions are satisfied:

(i) $u \in K, v \in F(u)+C, w \in G(u)+D$,

(ii) $(F, G)$ is generalized $C \times D$-convex at $\left(x_{0}, y_{0}, z_{0}\right)$ on $E$;

(iii) there exist $\phi \in C^{+i}$ and $\psi \in D^{*}$ such that

$$
\inf _{(y, z) \in \bar{\Theta}}\{\phi(y)+\psi(z)\}=0, \quad \psi\left(z_{0}\right)=0,
$$

$$
\text { where } \bar{\Theta}:=\bigcup_{x \in K} G-D^{b(2)}(F, G)\left(x_{0}, y_{0}, z_{0}, u-x_{0}, v-y_{0}, w-z_{0}\right)\left(x-x_{0}\right) \text {. }
$$

Then, the pair $\left(x_{0}, y_{0}\right)$ is a Benson proper efficient solution of (SOP).

\section{Acknowledgments}

The authors would like to thank anonymous referees for their valuable comments and suggestions, which helped to improve the paper. This research was partially supported by the National Natural Science Foundation of China (10901004, 11171362, and 11071267), Natural Science Foundation Project of CQ CSTC (cstcjjA00019), and Science and Technology Research Project of Chong Qing Municipal Education Commission (KJ100419).

\section{References}

[1] D.T. Luc, Theory of Vector Optimization, vol. 319, Springer, Berlin, Germany, 1989.

[2] J. Jahn, Vector Optimization, Springer, Berlin, Germany, 2004.

[3] J.-P. Aubin and H. Frankowska, Set-Valued Analysis, vol. 2, Birkhäuser Boston, Boston, Mass, USA, 1990.

[4] H. W. Corley, "Optimality conditions for maximizations of set-valued functions," Journal of Optimization Theory and Applications, vol. 58, no. 1, pp. 1-10, 1988.

[5] J. Jahn and R. Rauh, "Contingent epiderivatives and set-valued optimization," Mathematical Methods of Operations Research, vol. 46, no. 2, pp. 193-211, 1997.

[6] G. Y. Chen and J. Jahn, "Optimality conditions for set-valued optimization problems," Mathematical Methods of Operations Research, vol. 48, no. 2, pp. 187-200, 1998.

[7] J. Jahn and A. A. Khan, "Generalized contingent epiderivatives in set-valued optimization: optimality conditions," Numerical Functional Analysis and Optimization, vol. 23, no. 7-8, pp. 807-831, 2002.

[8] B. H. Sheng and S. Y. Liu, "On the generalized Fritz John optimality conditions of vector optimization with set-valued maps under Benson proper effeiciency," Applied Mathematics and Mechanics, vol. 23, no. 12, pp. 1289-1295, 2002.

[9] X.-H. Gong, H.-B. Dong, and S.-Y. Wang, "Optimality conditions for proper efficient solutions of vector set-valued optimization," Journal of Mathematical Analysis and Applications, vol. 284, no. 1, pp. 332350, 2003.

[10] L. Rodríguez-Marín and M. Sama, "About contingent epiderivatives," Journal of Mathematical Analysis and Applications, vol. 327, no. 2, pp. 745-762, 2007.

[11] C. S. Lalitha and R. Arora, "Weak Clarke epiderivative in set-valued optimization," Journal of Mathematical Analysis and Applications, vol. 342, no. 1, pp. 704-714, 2008.

[12] J. Jahn, A. A. Khan, and P. Zeilinger, "Second-order optimality conditions in set optimization," Journal of Optimization Theory and Applications, vol. 125, no. 2, pp. 331-347, 2005.

[13] S. J. Li, K. L. Teo, and X. Q. Yang, "Higher-order optimality conditions for set-valued optimization," Journal of Optimization Theory and Applications, vol. 137, no. 3, pp. 533-553, 2008.

[14] S. J. Li, K. L. Teo, and X. Q. Yang, "Higher-order Mond-Weir duality for set-valued optimization," Journal of Computational and Applied Mathematics, vol. 217, no. 2, pp. 339-349, 2008. 
[15] S. J. Li and C. R. Chen, "Higher order optimality conditions for Henig efficient solutions in set-valued optimization," Journal of Mathematical Analysis and Applications, vol. 323, no. 2, pp. 1184-1200, 2006.

[16] C. R. Chen, S. J. Li, and K. L. Teo, "Higher order weak epiderivatives and applications to duality and optimality conditions," Computers \& Mathematics with Applications, vol. 57, no. 8, pp. 1389-1399, 2009.

[17] Q. L. Wang and S. J. Li, "Generalized higher-order optimality conditions for set-valued optimization under Henig efficiency," Numerical Functional Analysis and Optimization, vol. 30, no. 7-8, pp. 849-869, 2009.

[18] Q. L. Wang, S. J. Li, and K. L. Teo, “Higher-order optimality conditions for weakly efficient solutions in nonconvex set-valued optimization," Optimization Letters, vol. 4, no. 3, pp. 425-437, 2010.

[19] J. Borwein, "Multivalued convexity and optimization: a unified approach to inequality and equality constraints," Mathematical Programming, vol. 13, no. 2, pp. 183-199, 1977.

[20] Q. L. Wang, "Erratum for "Higher-order generalized applications to duality of setvalued optimization"," Journal of Inequalities and Applications, vol. 2011, Article ID 817965, 3 pages, 2011.

[21] J. M. Borwein and D. Zhuang, "Super efficiency in vector optimization," Transactions of the American Mathematical Society, vol. 338, no. 1, pp. 105-122, 1993.

[22] J. Borwein, "Proper efficient points for maximizations with respect to cones," SIAM Journal on Control and Optimization, vol. 15, no. 1, pp. 57-63, 1977.

[23] G. Y. Chen and W. D. Rong, "Characterizations of the Benson proper efficiency for nonconvex vector optimization," Journal of Optimization Theory and Applications, vol. 98, no. 2, pp. 365-384, 1998.

[24] B. Jiménez and V. Novo, "Second order necessary conditions in set constrained differentiable vector optimization," Mathematical Methods of Operations Research, vol. 58, no. 2, pp. 299-317, 2003. 


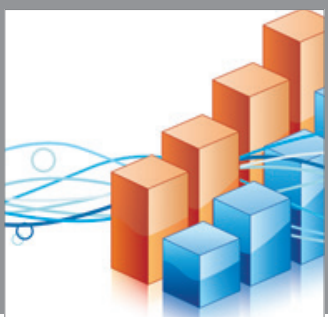

Advances in

Operations Research

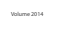

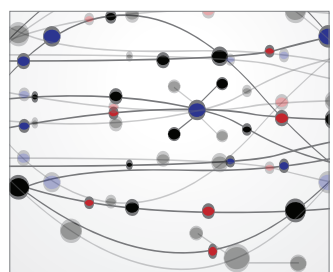

\section{The Scientific} World Journal
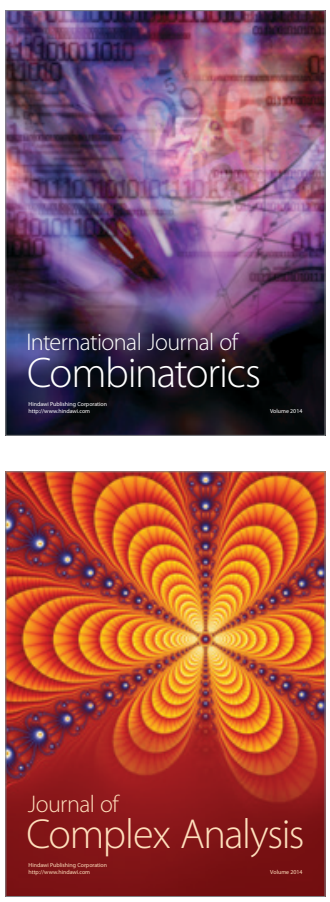

International Journal of

Mathematics and

Mathematical

Sciences
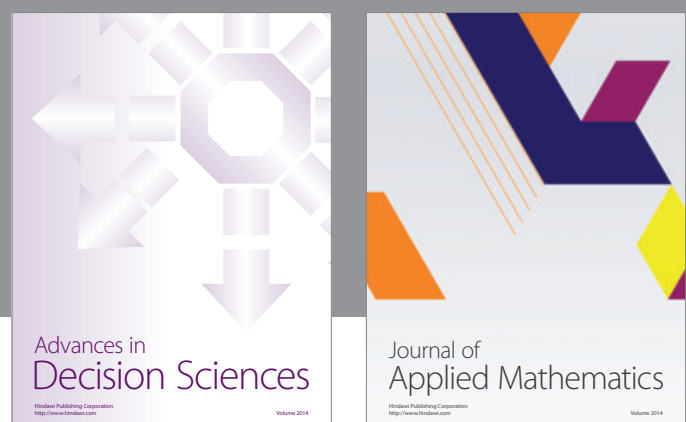

Journal of

Applied Mathematics
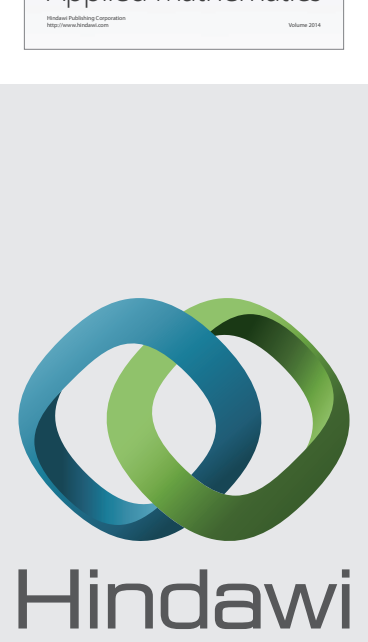

Submit your manuscripts at http://www.hindawi.com
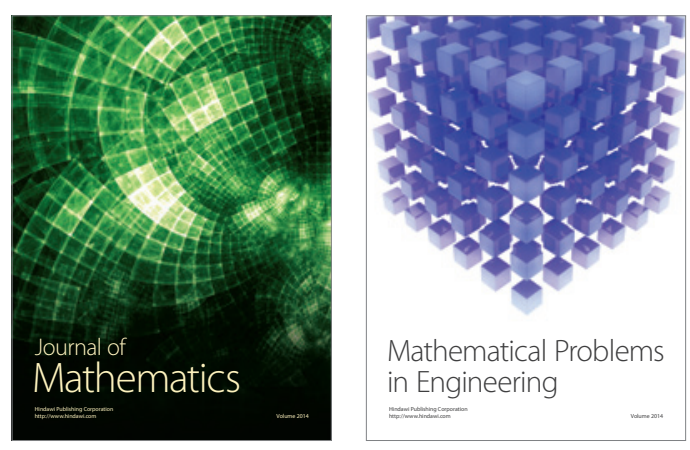

Mathematical Problems in Engineering
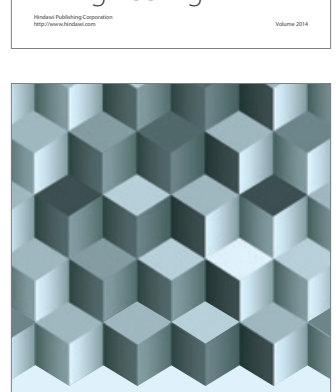

Journal of

Function Spaces
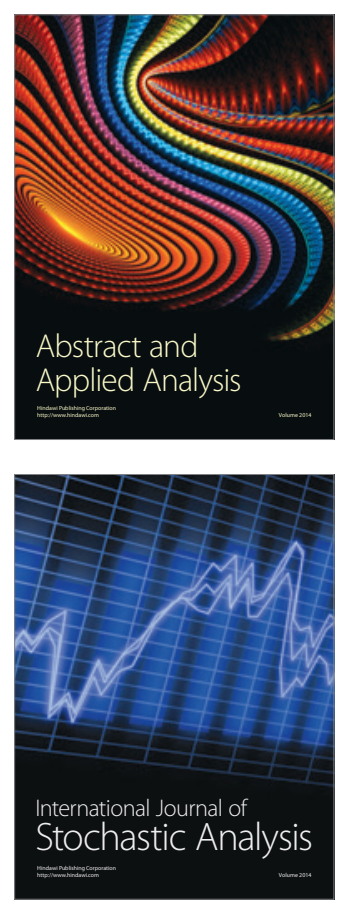

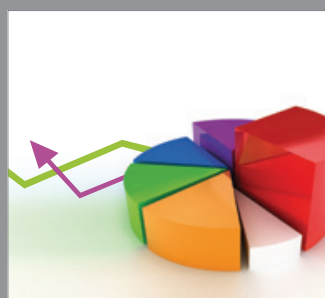

ournal of

Probability and Statistics

Promensencen
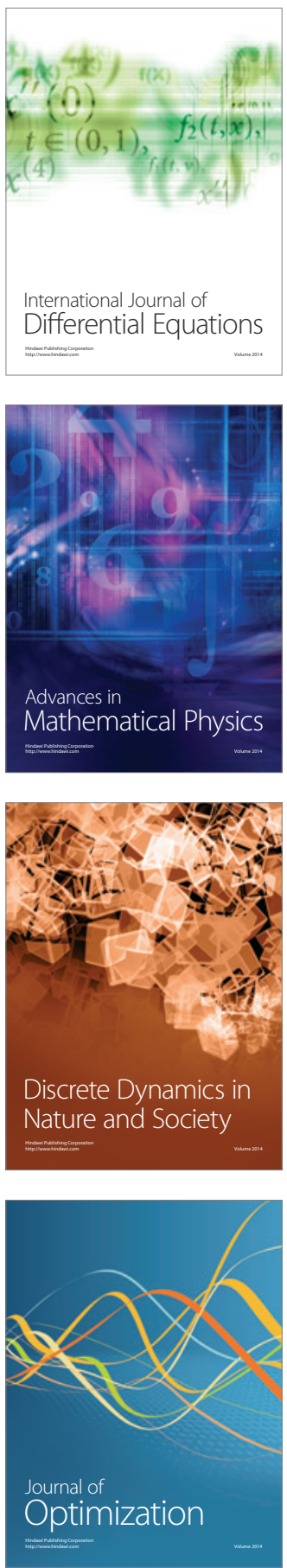